\section{TRAF3 mutations in canine B-cell lymphoma}

\section{Patrick Smith, Elspeth Waugh, Ruth Jarrett, Joanna Morris}

University of Glasgow, Glasgow, United Kingdom

\section{OBJECTIVES}

TRAF3 protein is an important negative regulator of the nuclear factor-kappaB pathway. Mutations of the TRAF3 gene are likely to be important in canine and human B-cell lymphoma, however only limited work has been done to characterise these mutations. This study aimed to investigate the prevalence and characteristics of TRAF3 mutations in canine B-cell lymphoma, and predict their functional impact.

\section{METHODS}

DNA was extracted from 24 B-cell lymphoma samples and 25 control lymph node samples (4 T-cell lymphomas, 21 reactive lymph nodes). $10 \mathrm{~B}$-cell lymphoma samples had non-neoplastic control DNA from peripheral blood cells. Exons 10 and 11 of the TRAF3 gene were amplified by PCR and Sanger sequenced. The data were analysed for potential mutations and the resulting impact on amino acid sequence was determined.

\section{RESULTS}

$6 / 24(25 \%)$ of B-cell lymphoma samples had mutations (two in exon 10, four in exon 11) predicted to truncate the TRAF3 protein and lead to loss of function. No T-cell lymphoma or reactive lymphoid tissue samples had functionally significant mutations. Two lymphomas with mutations had control tissue and in one case the mutation was identified as somatic. Two previously unidentified single nucleotide polymorphisms were identified in $11 / 49(22 \%)$ samples, $7 / 11(64 \%)$ of which had T-cell or B-cell lymphoma.

\section{STATEMENT (CONCLUSIONS)}

We showed inactivating mutations of TRAF3 are common in canine B-cell lymphoma, indicating loss of TRAF3 function may be important in canine B-cell lymphoma pathogenesis. Future studies assessing the prognostic and therapeutic value of TRAF3 mutation status are needed.

\section{Matrical carcinomas - a case series of four dogs}

\section{Giulia Tosi, Katie McNaught, Joanna S. Morris, Angelika F. Rupp, Jenny R. Helm}

University of Glasgow, School of Veterinary Medicine, Glasgow, United Kingdom

\section{OBJECTIVES}

Matrical carcinomas are a group of rare malignant follicular skin neoplasms comprising malignant pilomatricomas and malignant trichoepitheliomas. Whilst little is reported on the gold standard treatment of such masses in veterinary medicine, the prognosis of affected dogs appears to be poor. The aim of this study was to describe treatment and outcome of four cases of matrical carcinoma.

\section{METHODS}

Retrospective evaluation of clinical records for dogs presented to a referral hospital between January 2011 and
January 2019 identified four dogs that had been treated for histopathologically confirmed matrical carcinoma. Histopathology review was performed on all original biopsies

\section{RESULTS}

All dogs were initially treated surgically. Two dogs received adjuvant injectable carboplatin whilst a third dog, which had evidence of lymph node metastasis at presentation, was treated with combined radiotherapy and carboplatin chemotherapy. A fourth dog was treated with radiotherapy and metronomic chemotherapy to address gross pulmonary metastatic disease. This dog was euthanised 187 days after diagnosis due to progressive disease whilst the other three dogs are still alive (October 2019) and have not developed metastatic disease. The median follow up time for all patients was 349 days and ranged from 187 to 2940 days.

\section{STATEMENT (CONCLUSIONS)}

The prognosis for dogs without evidence of pulmonary metastatic disease on presentation seems considerably better compared to previous literature. Further studies are required to determine how treatment with radiotherapy and chemotherapy influence survival times for patients affected by matrical carcinomas. 